Authors' Reply

\section{Reply to Comment on: Weets I, Kaufman L, Van der Auwera B et al. (2004) Seasonality in clinical onset of Type 1 diabetes in Belgian patients above the age of $\mathbf{1 0}$ is restricted to HLA DQ2/DQ8-negative males, which explains the male to female excess in incidence. Diabetologia 47:614-621}

To the Editor: We thank you for your interest in our work [1] and the suggestions made.

Although we listed vitamin D status as one of many seasondependent external factors that possibly contribute to seasonality at diagnosis of Type 1 diabetes, our recent study [1] did not specifically aim at further elucidating the putative implication of vitamin $\mathrm{D}$ deficiency in the aetiology of Type 1 diabetes. We previously found no differences in season at birth between our group of Type 1 diabetic patients and the Belgian background population [2]. Also, there was no significant seasonality at diagnosis under age 10 [1]. Therefore our studies

DOI 10.1007/s00125-004-1540-2

Received: 3 June 2004 / Accepted: 28 July 2004

Published online: 22 October 2004

(C) Springer-Verlag 2004 provide no indirect evidence that putative season-dependent risk factors such as maternal, perinatal or childhood vitamin D deficiency play a central role in the initiation or precipitation of childhood-onset Type 1 diabetes.

However, these observations do not rule out vitamin D deficiency as one of the disease initiators or accelerators and further studies on the possible implication of vitamin D status and receptor polymorphisms in the pathogenesis of Type 1 diabetes are certainly warranted. In this respect, prospective studies in risk groups such as first-degree relatives, preferably followed from birth on, are likely to be most informative.

F. K. Gorus, I. Weets

Diabetes Research Center, Brussels Free University (VUB), Brussels, Belgium

\section{References}

1. Weets I, Kaufman L, Van der Auwera B et al. (2004) Seasonality in clinical onset of Type 1 diabetes in Belgian patients above the age of 10 is restricted to $H L A Q 2 / D Q 8$ negative males, which explains the male to female excess in incidence. Diabetologia 47:614-621

2. Weets I (2004) Etiological and phenotypical heterogeneity of diabetes presenting before age 40 according to demographic and biological parameters: variable contributions of genetic and external acceleration. $\mathrm{PhD}$ Thesis, Brussels Free University, Brussels, p 181

F. K. Gorus ( )

Diabetes Research Center, Brussels Free University (VUB),

Laarbeeklaan 103, 1090 Brussels, Belgium

E-mail: frans.gorus@az.vub.ac.be

Tel.: +32-2-4775030, Fax: +32-2-4775047 